\title{
A Clinical Experience of Ectopic Pregnancy and Allied Conditions.*
}

By F. W. N. Hatltain, M.D., F.R.C.P. (Edin.), Physician, Royal Maternity Hospital, Edinburgh; Lecturer on Obstetrics, Edinburgh School of Medicine.

There is no more perfect elinical picture than that portrayed by a typical case of ectopic pregnancy. Amenorrhœa followed by irregular hæmorrhages, discomfort in one or other side culminating in sudden intense abdominal pain with more or less shock and the shedding of a uterine cast. With such a clinical story, he who runs may read. The diagnosis hardly requires the detection of physical signs. On the other hand, in certain cases the symptoms are so indefinite and the history is so involved, that the interrogation of the patient is absolutely valueless, while the physical signs may be so closely simulated by other conditions that even to the expert the certain diagnosis is not only difficult, but almost impossible.

Having had a considerable, though by no means large, experience of such cases, I have presumed to lay it before the Fellows of the Society, in the hope that it may perhaps help to show what difficulties may assert themselves, and by these being discussed, tend to elucidate the diagnosis of this extremely important and dangerous complication of pregnancy.

My operative experience is drawn from 21 cases treated shortly after the occurrence of anxious symptoms, and 8 operated on at a remote period after the acute symptoms had passed off. In 3 of the latter it is only fair to state that the diagnosis was made from the history alone; microscopic evidence being either negative or unattainable. In another case, as will be described, no operation was performed. Thirty supposed cases in all, therefore, may be said to form my entire practical experience. Of the first group of 21 cases three were wrongly diagnosed and were not extra-uterine pregnancies, so that the actual number of verified ectopic gestations is diminished to 23 .

\section{Diagnosis.}

Broadly speaking the characteristic symptoms of ectopic pregnancy, before so-called rupture, may be said to bo four:--

\footnotetext{
* Read before the Edinburgh Obstetrical Society, Mar. 14, 1906.
} 
(1) A variable period of amenorrhcea.

(2) Irregular uterine hæmorrhage.

(3) Pelvic pain and discomfort.

(4) Shedding of uterine decidua.

The association of these go to form, as has already been shown, a typical case.

In the twenty-three cases under my observation where the history was carefully taken

$\begin{array}{lllllr}\text { Amenorrhœa was absent in... } & \ldots & \ldots & \ldots & \ldots & 5 \\ \text { Irregular hæmorrhage was absent in... } & \ldots & \ldots & 4 \\ \text { Pelvic pain or discomfort was absent in } & \ldots & \ldots & 4 \\ \text { Shedding of decidua was absent in } \ldots & \ldots & \ldots & 16\end{array}$

The most constant symptoms were, therefore, pelvic discomfort and irregular bleedings, and, the least constant, expulsion of a decidua. In fact this occurred so seldom that so far as my experience is concerned, its absence is of no moment in the diagnosis.

In two instances, before the severe symptoms of intraperitoneal hæmorrhage occurred there was no evidence of anything beyond a normal pregnancy; amenorrhoea was absolute, and the patient in no way complained of pelvic discomfort. One of these progressed to the 12 th week, and the second to the 8 th week. In the latter, in spite of symptoms suggestive of intraperitoneal bleeding, the gestation was allowed to progress to the 10 th week, when a severe hæmorrhage from secondary rupture called for immediate laparotomy, Amenorrhoa was absolute throughout. It will thus be evident from the history that individual cases may show little or nothing before the actually dangerous symptoms point to the serious nature of the condition, while, as a rule, even should the classic symptoms be present, they are frequently considered by the patient to be of such slight consequence that medical advice is but seldom taken. Hence the rarity with which an actively growing ectopic gestation is met with before dangerous complications occur.

Perhaps the symptom above all others which gives rise to apprehension on the part of the patient is the frequent and irregular occurence of hæmorrhage either with or without previous amenorrhcea. This leads one to consider what is the actual cause of the bleeding. Is it purely uterine, or is it tubal from the separation of the ovum? It cannot but be maintained that the attachment of the ovum to the tube is in the vast majority of cases feeble, and the tendency of all ectopic pregnancies is towards early separation, bleeding, and death of the ovum. Hence the frequency 
- Marks seat of xupture.

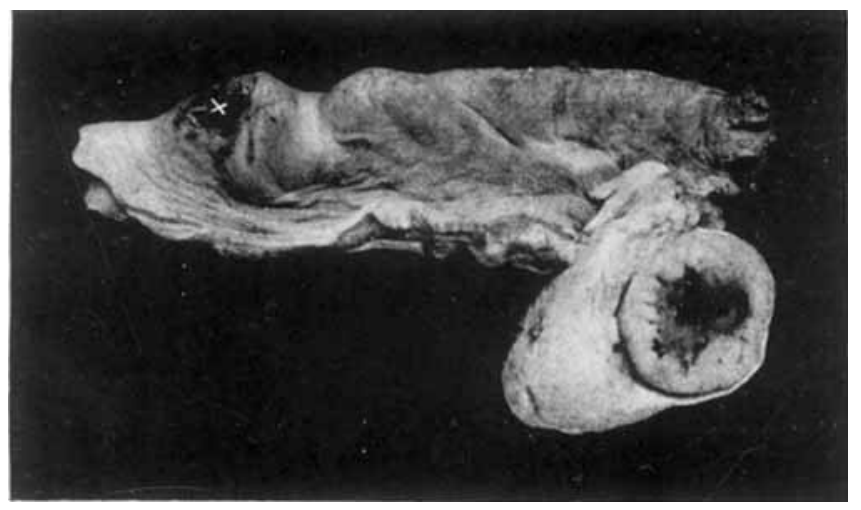

NATURAL SIZE.

Fia. 1.-Fiarly Tubal Pregnancy removed for ruplure, associated with sovere intraporitoneal bleeding and collapse after 10 days' amenorrhoea. 


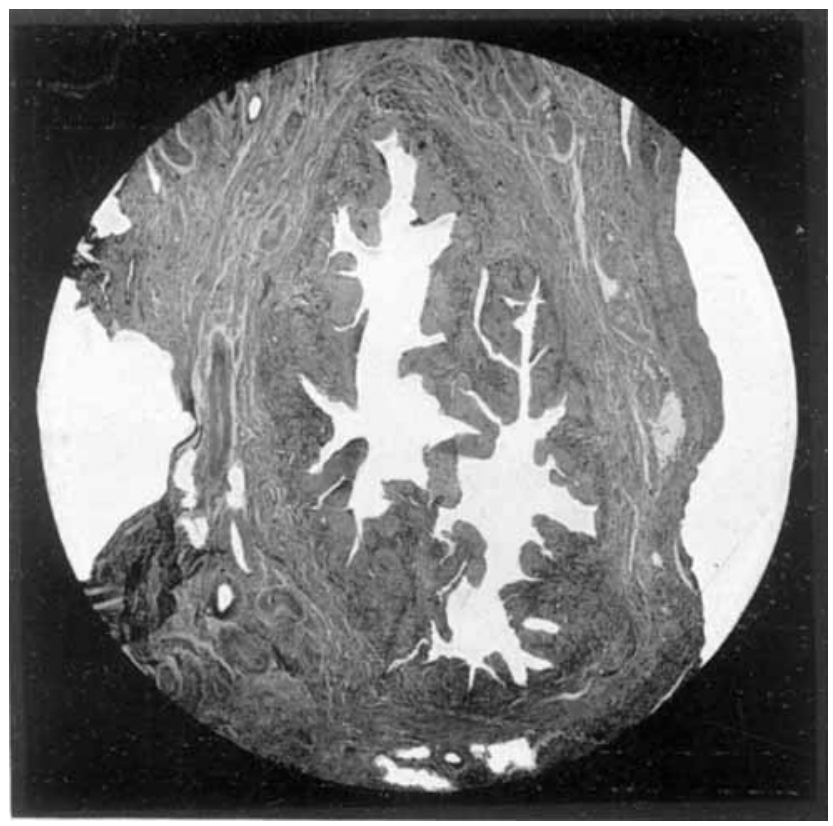

Fis. 2.-Section of opposite Tube from I'ubal Gestalion. showing Decidual formation in nucosa $\times 8$ diems. 


\section{Haultain: Ectopic Pregnancy}

of tubal mole. This is probably due to the absence of a tubal decidua in the majority of cases. In some, however, there can be no doubt of the formation of a decidua, as I have verified in two of the cases I have microscopically examined, and, in one case in which I removed the uterus and opposite tube for a ruptured gestation sac, the opposite tube, though apparently normal to the naked eye, was found to be lined by a typical decidua from end to end. As is shown by the accompanying microphotographs this is divided into a distinct spongy and compact layer. (See Figs. 2, 3 and 4.)

On separation of the ovum, bleeding is the necessary sequence, and the escape of blood must be looked upon as more or less salutary, for should the blood be retained, rupture of the thinned tube must almost inevitably follow. Through its escape, however, excessive tension on the thin tubal wall is averted, and rupture avoided. The direction of the blood flow will necessarily follow in the line of least resistance, and will thus pass either inwards towards the uterine cavity, and give rise to irregular hæmorrhages, or outwards through the abdominal ostium into the peritoneal cavity and occasion abdominal symptoms. When an extensive separation occurs it is probable that the blood will pass in both directions, hence the association of vaginal hæmorrhages at the time of the severe symptoms, due to the intraperitoneal bleeding. One may therefore expect, as is confirmed by observation, that in some instances the bleeding may proceed entirely in one or other direction, and death of the ovum simultaneously result from separation. This will account, on the one hand, for these cases of tubal mole without serious symptoms having manifested themselves, as the bleeding occurs only from the vagina, and on the other hand for intraperitoneal hæmorrhage without vaginal bleeding.

Further, it is possible that the rapid distension of the tube in these cases may give rise to the severe pain in the side, which is such a constant symptom at the time of marked separation. In ectopic gestation hæmorrhage from the vagina or into the peritoneal cavity through the abdominal ostium might fitly be termed "threatened tubal abortion," as it in every way coincides with the intrauterine condition known as "threatened abortion." Shedding of the decidua probably results after the death of the ovum.

As will be noted from the table quoted, shedding of the decidua only occurred in four of the cases operated upon. This may be considered as due to the fact that the operation was undertaken before the change necessary for the separation of the decidua had taken place, or that it had come away unnoticed. It is probable that 
the uterine decidua is not shed in the majority of cases until some time after the death of the ovum. This explains its expulsion in some cases after the gestation sac has been removed by operation. In one such case the bleeding which attended it on the night after the operation nearly caused the death of the already exsanguine woman, and had to be arrested by vaginal plugging. This is surely a variety of post partum hæmorrhage not hitherto described.

Intraperitoneal escape of blood through the abdominal ostium has been shown by Cullingworth and others to be by far the most frequent cause of urgent symptoms; in this $\mathrm{my}$ experience entirely coincides. Of the 21 cases I have operated upon, in only six has the actual tubal rupture been found. In only one of these has the condition been met with before the 9 th week. This exception, however, is sufficiently striking to be worthy of detailed description:-After ten days' amenorrhœa the patient was suddenly seized with the usual acute symptoms of intraperitoneal bleeding, and in the course of five hours was reduced to such an exsanguine state that the pulse was almost imperceptible at the wrist. As the specimen shows, the gestation was about the size of a pea and was situated in the ampullary portion of the tube. The bleeding in this instance seemed to be due to the perforation of a large vein.

\section{FIG. I.}

The differential diagnosis between intra-peritoneal bleeding from actual rupture from the sac as opposed to escape from the ostium of the tube is mainly to be based upon constitutional signs, particularly the temperature. The physical signs which develop from the ostial trickle consist in sudden severe hypogastric pain, particularly referable to the side, marked tenderness of the abdomen, and an increased temperature. While in cases of rupture where the bleeding is naturally more severe the symptoms of pain are associated with a distinct fall in the body heat, and more evidence of shock. In these cases I have noted at the same time the patient complained of marked epigastric tenderness and discomfort.

Of course, in cases where the bleeding from the abdominal ostium is excessively severe the same symptoms will appear, but such a condition is quite the exception. As a rule, bleeding through the ostium is limited in amount and seems to be spontaneously checked from a resulting local peritonitis by which the effused blood is encapsulated. A definite pelvic swelling can therefore in the course of some hours be mapped out. If large, this is usually retro-uterine and dislocates the uterus forwards in front of it. The acute symp- 


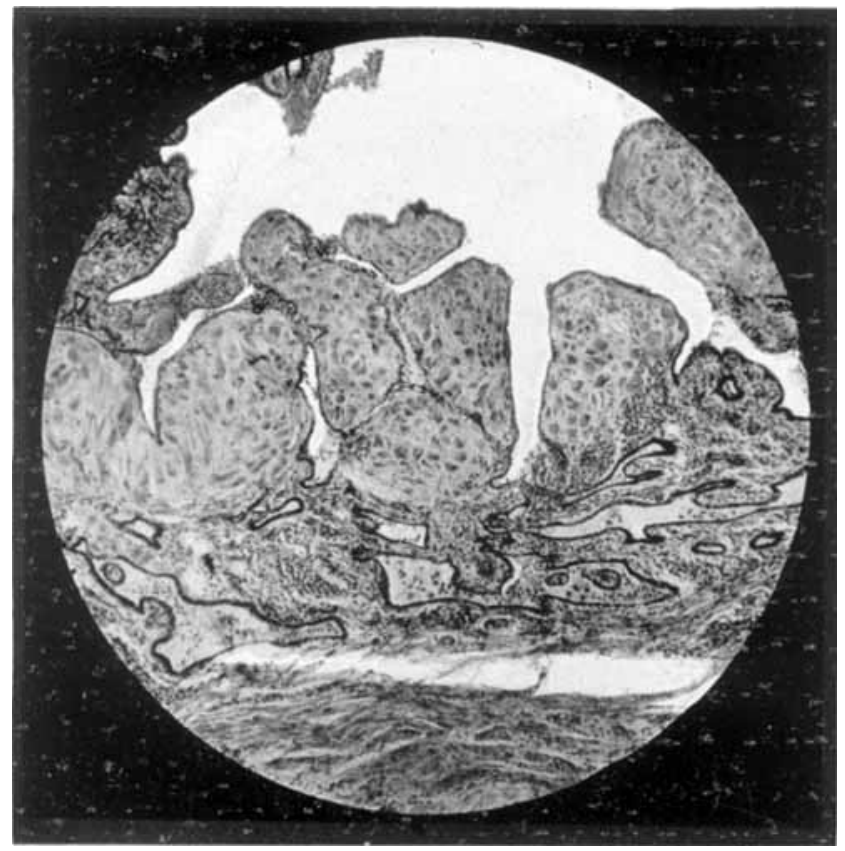

Frc. 3.-.Tubal Decidna. Opposile Tube from. Tubal Gestation, showing spongy and eompact layers $\times$ 60. 


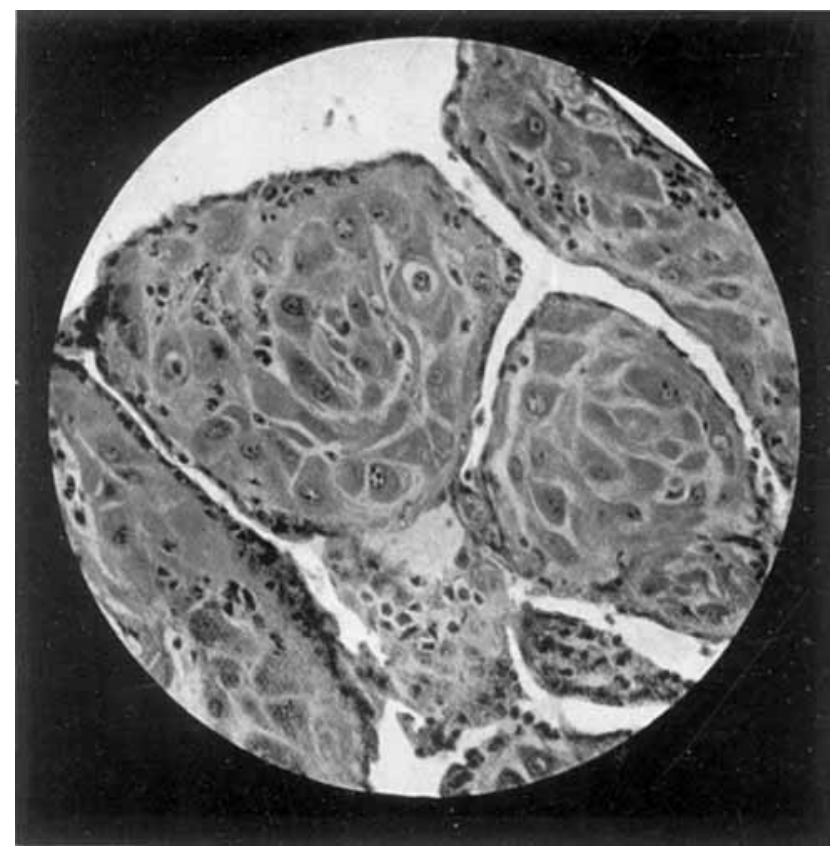

Frg. 4.- Trubal Decidua. Opposite Tube from T'ulual Pregnancy, showing decidual cells, and low eubical epithelinu $\times 200$ dia. 
toms having passed off, the patient soon returns to her previous state of health, only, in the majority of cases, to be subjected to a recurrence of similar symptoms from renewed extravasation of blood. The rapid return of the patient to a feeling of well-being, and the absence of abdominal tenderness with the completely normal state of the pulse and temperature, frequently throw the medical attendant off his guard and lead him to temporise until another and perhaps severer intraperitoneal hæmorrhage develops. Should death of the ovum and complete separation occur, the hæmorrhage may permanently cease and the blood be absorbed, giving rise to the cases of so-called complete recovery. In these cases, however, unless the ovum has itself been expelled, it must remain as a foreign body in the Fallopian tube (tubal mole). In exceptional instances, when very small, entire absorption seems to be possible and the tube is restored to its original patent state. Usually, however, a permanent occlusion results (hæmatosalpinx), which frequently gives rise to prolonged symptoms of pelvic discomfort, and occasionally from infection becomes the seat of grave pyogenic mischief.

In the same way blood effused into the peritoneal cavity may be absorbed or remain as an eucapsulated mass, which may subsequently be infected and form a pelvic abscess, or give rise to much discomfort from the formation of adhesions to the uterus and its adnexa, or to the surrounding viscera.

\section{Differential diagnosis.}

As may be inferred from what has already been stated regarding the absence of a typical history, the certain diagnosis of ectopic pregnancy is frequently one of the greatest difficulty, indeed it is a question if it is possible, as it may be simulated by other conditions in which the physical signs are identical. It would be invidious in a short paper to pretend to consider the many conditions with which it had been confounded. I shall therefore confine myself to the record of mistakes in my own experience.

These may be divided into first, cases operated upon in which I expected to find an extra-uterine gestation, secondly, a case diagnosed otherwise in which ectopic pregnancy was found.

(1) Mrs. H., 35; menstruation irregular; was suddenly seized with acute abdominal pain and with faintness. She showed the signs of concealed hæmorrhage. The abdomen was tender on pressure, and on pelvic examination the uterus was felt to be somewhat enlarged, and to the right there was a swelling the size of a turkey's egg. On opening the abdomen the cavity was found filled with blood, and, on 
the right side of the uterus, a dermoid ovarian cyst, with a ruptured vein in the pedicle.

(2) Mrs. P., aged 27. After 5 weeks' amenorrhoa complained of great pelvic discomfort. On bimanual examination the uterus was found enlarged and pushed forward by a soft swelling, which, when laparotomy was performed, proved to be a simple unilocular ovarian cyst associated with pregnancy.

(3) Mrs. K., aged 40, complained of profuse irregular vaginal bleeding which had lasted for 10 months, and of having recently suffered from retention of urine necessitating the occasional use of the catheter. A large hard swelling was found in the retro-uterine pouch which pushed the uterus forward. Incarcerated fibromyoma was diagnosed, but on operation a large tense blood sac was found enclosing an atrophied ovum.

(4) Mrs. B., aged 30, complained of constantly recurring vaginal hæmorrhage, which had lasted for 3 months after a menstrual period delayed a few days. There was a history of persistent pain in left side with severe exacerbations. Temperature normal-pulse under 100. On vaginal examination a soft, tender, pulsating, cystic swelling was felt to the left of the uterus about oqual in size to a turkey's egg. I considered it to be a tubal mole, but found on opening the abdomen an ovarian abscess and salpingitis.

A case which came under my notice with the late Dr. Milne Murray is perhaps interesting in this connection, although no operation was performed, and may be recorded:-

Mrs. S., aged 26 , after 2 months' amenorrhoa expelled from the vagina a distinct uterine decidual cast, associated with much pain and hæmorrhage. Five weeks afterwards she complained of severe pelvic discomfort. On examination a swelling equal in size to a small cocoanut was found in the pelvis, and to the left a body which appeared to be the uterus could be distinctly felt, and into which a sound was passed over four inches. In the course of examination it was felt that the swelling changed markedly in consistence, which suggested an intra-uterine pregnancy. Three months afterwards she expelled a six months' ovum, and on subsequent bimanual examination a bicornute uterus was distinctly made out.

Another case which fortunately I did not operate on is of much interest:- -

Mrs. McL., age 30. Married 5 years; no children; complained of being seized with intense abdominal pain. She gave a history of two months' amenorrhœa, followed by severe almost persistent vaginal hæmorrhage for the last three weeks. Bowels irregular-constipation 


\section{Haultain: Ectopic Pregnancy}

alternating with diarrhœa. On palpation an excecdingly tender swelling was found to the right of the middle line in the hypogastric region, which $I$ took to be the gravid uterus pushed over by a tonse, ill-defined mass, on the left side, over which there was also considerable tenderness on pressure. From the sudden onset of the severe pain I diagnosed a combined intra-uterine and tubal pregnancy, and determined to operate. In the usual preparation for operation a large dose of castor oil was given, with the result that the left-sided swelling rapidly subsided, and the patient was restored to complete health.

From the foregoing cases it will be evident that the differential diagnosis may, in certain instances, be one of the utmost difficulty, and it is almost impossible to see how mistakes can be avoided. Other similar cases might be cited, such as the difficulties which may occur in differentiating gravid retroversion, and the co-existence of fibroids and pregnancy. But perbaps the most common condition with which it may be confounded is a simple early abortion. The presence of the irregular and profuse hæmorrhages which occur after a short period of amenorrhoea, with the painful expulsion of a uterine cast may readily bo considered an ordinary abortion, if no thorough examination be made of the pelvic organs. Further, in many instances, even though no decidual east has been noted to be expelled, the diagnosis of simple abortion is most readily come to from its comparative frequency; and it is only after scvere symptoms of intraperitoneal homorrhage have occurred, that the true condition of affairs is made apparent. Such being the case, it must be strongly urged that in evcry case of apparently early abortion the pelvis should be carefully examined, and mistakes thus avoided.

Perhaps one of the most striking results of my expcrience has been the frequency of recurrence of tubal pregnancy. In four cases this has shown itself. In one, after removal of a tubal gestation on the right side, I had to romove a similar condition on the left side within nine months. In a second, a recurrence took place in the opposite tube within cighteen months, and was removed by another operator. In a third, a distinct history of tubal gestation in the left tube was fully verified two years afterwards, when I operated for a ruptured sac on the right side. While the fourth was an identical case, the position of the gestation sac being reversed. Intraligamentary bleeding from rupture $I$ have met with only in two cases; in one I operated immediately on its occurrence; while in the other I was urgently called to operate for severe intraperitoneal bleeding, due to secondary rupture through the broad ligament. 
Treatment.

As regards the treatment of the condition, increasing experience convinces me more and more that it resolves itself into removal in all cases. A tubal pregnancy must be considered in the light of a malignant growth, and a continued menace to the life of the patient so long as it is present. To temporize in the fond hope that absorption and a spontaneous cure will occur is totally unworthy of consideration, as at the best one can hardly hope for a complete cure to be obtained. Occasionally, I admit, symptoms may entirely cease, but an oceluded tube must, in the majority of these cases, be the lasting result. In many instances, however, sudden and severe complications necessitate immediate interference under conditions which from their urgency are by no means as satisfactory as if removal had been adopted at an earlier stage. As I have previously stated, I have had occasion to operate on eight cases at a remote period after well-marked evidence had shown itself of the presence of tubal pregnancy. Three times for constant pelvic pain; twice for suppuration, and once for acute intra-peritoneal bleeding : all of which might have been avoided by prompt action when the condition was first discovered.

It is generally admitted when scvere intraperitoneal hæmorrhage is present immediate operation forms the patient's only chance. In cases where an intraperitoneal ooze of blood indicates its presence operative procedure need not be so immediate, but removal at the earliest convenient opportunity is demanded. In like manner the excision of a tubal pregnancy, if it should be diagnosed before symptoms of hæmorrhage have appeared, is essential.

Another strong point in favour of early operation is the ease and safety with which it can be accomplished, as the adhesions, if present, are few and easily separated, while in a later stage they may be extremely dense and difficult to manipulate. To put it succinctly, early operation is usually easy and safe, whilst it removes all chance of further trouble. To temporize on the bare chance of a complete cure by absorption is to expose the patient to definite risks of suppuration, continued growth of the gestation, and recurrent severe hæmorrhage, while the operation itself is frequently rendered one of the utmost difficulty.

As regards the modus operandi I have nothing original to suggest, but the method $I$ have adopted in recent cases is to ligature the infundibulo-pelvic and uterine ends of the sae, which I subsequently enucleate from between the layers of the broad ligament, and stitch the latter together by a fine continuous suture. In old- 


\section{Haultain: Ectopic Pregnancy}

standing cases, where a hæmatocele has been shut off by strong adhesions, or where the blood clot is enclosed between the layers of the broad ligament, after enucleation of the sac and surrounding blood clot, I pack the cavity with sterilized gaure, the end of which is pushed through an opening in the fornix vagince. By this means sufficient drainage is afforded, while at the same time the troublesome venous ooze is stooped. As far as possible, the cavity with the packing is shut off from the peritoneum by bringing the serous edges of the sac together by fine sutures.

Amongst the cases mentioned I have unfortunately to record four deaths. In only one of these, however, was the operation undertaken shortly after the onset of symptoms. In this instance an early interstitial tubal rupture had reduced the patient to the utmost limits of exsanguinity, from which she never rallied. The remaining three operations were, in two instances, performed for secondary peritonitis, one of these was purulent and almost hopeless from the start. The fourth death resulted from a case in which secondary rupture had occurred several weeks after the initial signs of intraperitoneal bleeding. From an experience such as this it can hardly be wondered how strongly I am opposed to anything but immediate removal, when even the probable diagnosis of the presence of tubal gestation is made.

To summarize the chief points which I have attempted to bring out, and which I should like to emphasize, are:-

(1) The difficulty of diagnosis from the irregularity of the signs and symptoms.

(2) The frequency with which it is simulated by other conditions.

(3) The ease with which it may be mistaken for an ordinary abortion, and the necessity for thorough pelvic examination in all such cases.

(4) And lastly, but most urgently, the absolute necessity for removal as soon as even a probable diagnosis has been made. 